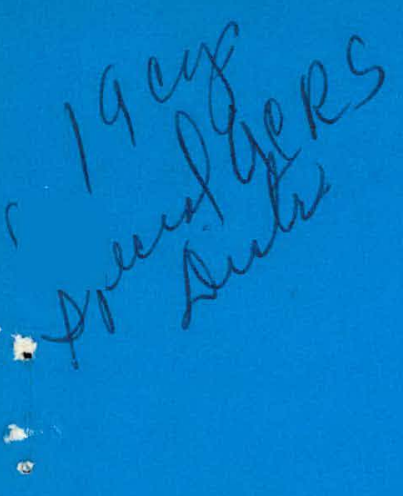

1973.

STEADY TEMPERATURE DISTRIBUTIONS IN THE FAR-FIELD REGION OBTAINED BY SOLUTION OF THE EQUATION OF CONVECTIVE DIFFUSION IN TWO DIMENSIONS Lawrence Dresner 


\section{DISCLAIMER}

This report was prepared as an account of work sponsored by an agency of the United States Government. Neither the United States Government nor any agency Thereof, nor any of their employees, makes any warranty, express or implied, or assumes any legal liability or responsibility for the accuracy, completeness, or usefulness of any information, apparatus, product, or process disclosed, or represents that its use would not infringe privately owned rights. Reference herein to any specific commercial product, process, or service by trade name, trademark, manufacturer, or otherwise does not necessarily constitute or imply its endorsement, recommendation, or favoring by the United States Government or any agency thereof. The views and opinions of authors expressed herein do not necessarily state or reflect those of the United States Government or any agency thereof. 


\section{DISCLAIMER}

Portions of this document may be illegible in electronic image products. Images are produced from the best available original document. 
This report was prepared as an account of work sponsored by the United States Government. Neither the United States nor the United States Atomic Energy Commission, nor any of their employees, nor any of their contractors, subcontractors, or their employees, makes any warranty, express or implied, or assumes any legal liability or responsibility for the accuracy, completeness or usefulness of any information, apparatus, product or process disclosed, or represents that its use would not infringe privately owned rights. 
Contract No. W-7405-eng-26

REACTOR DIVISION

\section{STEADY TEMPERATURE DISTRIBUTIONS IN THE FAR-FIELD REGION OBTAINED BY SOLUTION OF THE EQUATION OF CONVECTIVE DIFFUSION IN TWO DIMENSIONS}

Lawrence Dresner

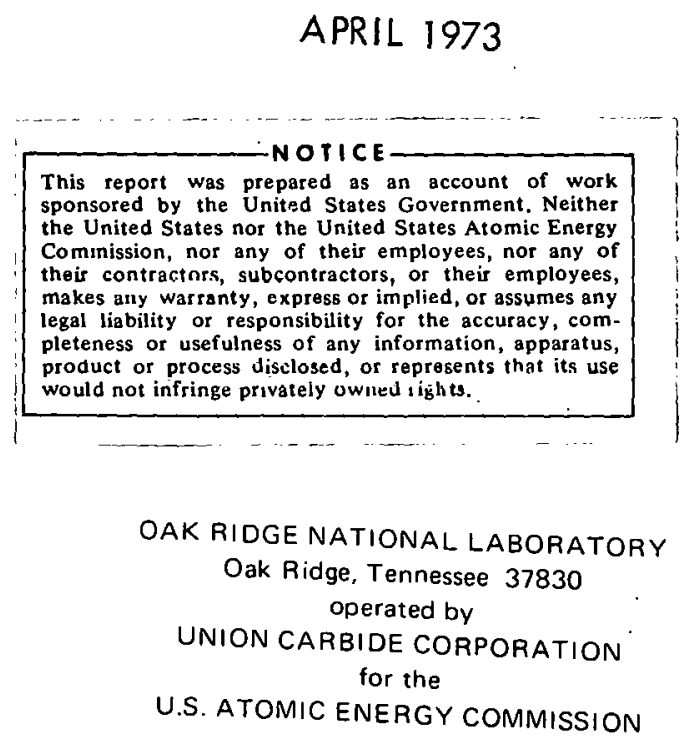




\section{THIS PAGE}

\section{WAS INTENTIONALLY \\ LEFT BLANK}




\section{CONTENTS}

Foreword $\ldots \ldots \ldots \ldots \ldots \ldots \ldots \ldots \ldots \ldots \ldots \ldots \ldots \ldots \ldots \ldots \ldots \ldots \ldots$

Abstract $\ldots \ldots \ldots \ldots \ldots \ldots \ldots \ldots \ldots \ldots \ldots \ldots \ldots \ldots \ldots \ldots \ldots \ldots \ldots \ldots \ldots \ldots \ldots$

Introduction $\ldots \ldots \ldots \ldots \ldots \ldots \ldots \ldots \ldots \ldots \ldots \ldots \ldots \ldots \ldots \ldots \ldots \ldots$

Basic Equations $\ldots \ldots \ldots \ldots \ldots \ldots \ldots \ldots \ldots \ldots \ldots \ldots \ldots \ldots \ldots \ldots \ldots \ldots \ldots$

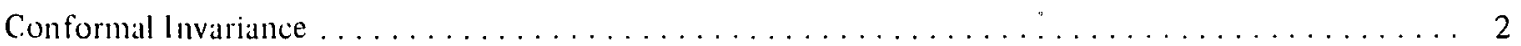

Point Source in a Uniformly Flowing, Infinite Medium $\ldots \ldots \ldots \ldots \ldots \ldots \ldots$

Use of the Velocity Potential and the Stream Function as Coordinates . . . . . . . . . . . 6

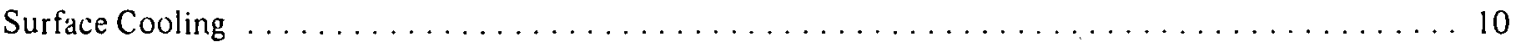

Ioining the Far-Field Solution to the Near-Field Solution $\ldots \ldots \ldots \ldots \ldots \ldots \ldots \ldots$

Half-Space with a Breakwater $\ldots \ldots \ldots \ldots \ldots \ldots \ldots \ldots \ldots \ldots \ldots \ldots \ldots \ldots \ldots$

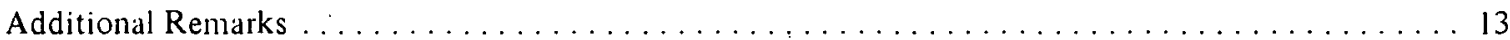

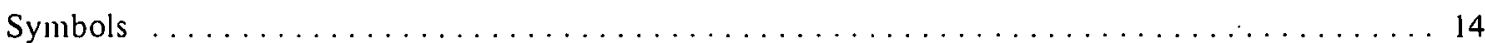

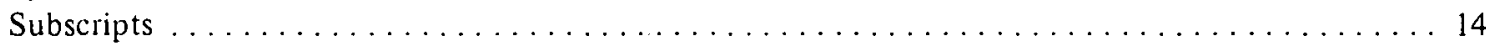

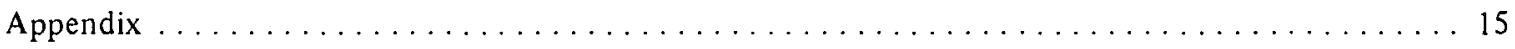


THIS PAGE

\section{WAS INTENTIONALLY LEFT BLANK}




\section{FOREWORD}

Oak Ridge Naltional Laboratory is providing technical assistanice to the U.S. A tomic Energy Commission Directorate of Licensing in its analyses of the environmental impact of power reactors as required by the National Envirommental Policy Act. The assessment of the environmental impact of a nuclear station has required the devclopment of new analytical techniques and the expansion of many existing ones.

This document is one of the spin-off benefits of this effort by the Commission and its National Liboratories to protect the environment. The work reported here, while originally completed in support of the analysis of a particular nuclear plant, has wide application in other areas. 


\title{
Steady Temperature Distributions in the Far-Field Region Obtained by Solution of the Equation of Convective Diffusion in Two Dimensions
}

\author{
Lawrence Dresner
}

\begin{abstract}
A mellow based on conformal mapping is suggested for calculating steady, far-ficld temperature distributions in two dimensions in an arbitrary potential flow field. Two numerical programs for performing the calculations are described and listed, and sample computations are given.
\end{abstract}

Keywords: diffusion, thermal pollution, heat transfer, convection, model, analytical model, computer program.

\section{INTRODUCTION}

A number of authors have used the equation of convective diffusion [Eq. (3) below] to calculate steady-state, far-field temperature rises caused by the release of waste heat into bodies of water. ("Far ficld" means that region in which transport by ambient turbulence is much greater than transport by turbulence generated by the discharge itself.) A short review of the literature on this subject may be found in the article of Harleman and Stolzenbach.' The problem has two aspects: (1) the determination of the appropriate values of the eddy diffusivities and the velocity components and (2) the solution of the equation of convective diffusion for the temperature field. Edinger and Polk ${ }^{2}$ and $\mathrm{C}_{\text {sanady }}{ }^{3}$ considered the second aspect under the assumption that the eddy diffusivities and the velocity components were constant and gave simple analytic solutions to the equation of convective diffusion. Both sets of authors ignored longitudinal diffusion, that is, diffusion in the direction of the flow velocity. This report generalizes their results in the two-dimensional case to an arbitrary potential flow field and includes longitudinal diffusion as well. This latter inclusion is of little practical importance, since longitudinal diffusion is small except close to the source [see discussion following Eq. (24)] ; but it is of great theoretical importance, because without the longitudinal diffusion term, the equation of convective diffusion loses the all-important property of conformal invariance. The generalization to an arbitrary two-dimensional potential flow field, on the other hand. is of great practical importance, because situations may arise in which the flow field can hardly be considered uniform. Examples of such situations will be given later.

\section{BASIC EQUATIONS}

The heat flux $\mathrm{q}\left(\mathrm{cal} \mathrm{cm} \mathrm{cm}^{-2} \mathrm{sec}^{-1}\right.$ ) is related to the temperature gradient $\nabla T$ and the flow velocity $\mathrm{v}$ as follows:

$$
\mathbf{q}=-k \nabla T+\rho c_{p} \mathbf{v} T
$$

J. D. F. Harleman and K. D. Stolzenbach, "Fluid Mechanics of Heat Disposal," A nn. Rev..Fluid Mech. 4; M. vani Dyke, W. G. Vincenti, and J. V. Wehausen (eds.), Annual Reviews, Inc., Palo Alto, Calif., pp. 7-32, 1972.

2. J. E. Edinger and E. M. Polk, Jr., "Initial Mixing of Thermal Discharge into a Uniform Current," in Part 1 of Heated-Effluent Dispersion in Large Lakes; State-of-the-Art of Analytical Modeling, A. J. Policastro and J. V.. Tokar, ANL/ES-11, Argonne National Laboratory, Center for Environmental Studies, pp. 173-88 (January 1972).

3. G. T. Csanady, "Offshore Outfall and Shoreline Surface Discharge Models for the Prediction of Thermal-Plume Dispersion in the Great Lakes," in Part 1 of Heated-Effluent Dispersion in Large Lakes: State-of-the-Art of Analytical Modeling, A. J. Policastro and J. V. Tokar, ANL/ES-11, Argonne National Laboratory, Center for Environmental Studies, pp. 189-206 (January 1972). 
where $k$ is thermal conductivity (including the contribution from turbulent eddies and assumed constant) ( $\left.\mathrm{cal} \mathrm{cm}^{-1} \mathrm{sec}^{-1} \mathrm{deg}^{-1}\right), \rho$ is fluid density $\left(\mathrm{g} / \mathrm{cm}^{3}\right)$, and $c_{p}$ is heat capacity of the fluid (cal $\left.\mathrm{g}^{-1} \mathrm{deg}^{-1}\right)$. In steady state, a differential heat balance at any point can be written

$$
\nabla \cdot \mathrm{q}=S,
$$

where $S$ is heat source density (cal cm${ }^{-3} \sec ^{-1}$ ). Since $\nabla \cdot v=0$ in incompressible flow, Eqs. (1) and (2) can be combined to give

$$
-k \nabla^{2} T+\rho c_{p} v \cdot \nabla T=S
$$

A flow may be called two dimensional if, for example, the flow velocity $\mathbf{v}$ depends on only two coordinates, say $x$ and $y$, and not on the third coordinate $z$. If the heat source $S$ also depends only on $x$ and $y$ and not on $z$, and if the bounding surfaces are cylinders whose generators are parallel to the $z$ axis, then the temperature $T$ also depends only on $x$ and $y$ and not on $z$. Such a temperature field may also be called two dimensional. This report is concerned exclusively with such two-dimensional flow and temperature fields.

One very important heat source is a line parallel to the $z$ axis. The intersection of this source with any plane parallel to the $x, y$ plane is a point. Because we want to reserve the term "line source" for something else, we call such a source a point source after its intersection with the $x, y$ plane. Another important heat source is a segment of a plane parallel to the $z$ axis whose intersection with any plane parallel to the $x, y$ plane is a line segment. We call such a source a line source after its intersection with the $x, y$ plane.

A line source may be approximated by a closely spaced collection of point sources. If the point sources are of equal strengths, the line source is uniform. The point sources need not be of equal strengths, however, in which case the source strength of the line source is not uniform but is weighted in some way along the line. One important weighting is a Gaussian weighting, since the Gaussian distribution arises when we try to join a far-field solution to the near field created by a single jet.

\section{CONFORMAL INVARIANCE}

A most important property of Eq. (3) is its invariance to conformal transformations when $\mathbf{v}$ is the velocity of an incompressible, irrotational flow. A conformal transformation is a two-dimensional transformation from the coordinates $x, y$ to coordinates $\xi, \eta$ which obeys certain conditions. The transformation

$$
\begin{aligned}
& \xi=\xi(x, y), \\
& \eta=\eta(x, y),
\end{aligned}
$$

is conformal if

$$
\begin{aligned}
& \xi_{x}=\eta_{y}, \\
& \xi_{y}=-\eta_{x},
\end{aligned}
$$


where the subscripts denote partial differentiation. Equations ( $5 a$ and $5 b)$ are called the Cauchy-Riemann cquations. It follows from Eqs. (5) that

$$
\begin{aligned}
& \xi_{x x}+\xi_{y y}=0, \\
& \eta_{x x}+\eta_{y y}=0, \\
& \xi_{x} \eta_{x}+\xi_{y} \eta_{y}=0, \\
& \xi_{x}{ }^{2}+\xi_{y}{ }^{2}=\eta_{x}{ }^{2}+\eta_{y}{ }^{2} .
\end{aligned}
$$

Let us now consider any function $f$ of $x, y: f$ can be considered a function of $\xi, \eta$ as well because of Eqs. (4). Then, differentiating, we find

$$
\begin{aligned}
& f_{x}=f_{\xi} \xi_{x}+f_{\eta} \eta_{x}, \\
& f_{y}=f_{\xi} \xi_{y}+f_{\eta} \eta_{y} .
\end{aligned}
$$

Differentiating again, we get

$$
\begin{aligned}
& f_{x x}=\left(f_{\xi \xi} \xi_{x}+f_{\xi \eta} \eta_{x}\right) \xi_{x}+f_{\xi} \xi_{x x}+\left(f_{\eta \xi} \xi_{x}+f_{\eta \eta} \eta_{x}\right) \eta_{x}+f_{\eta} \eta_{x x}, \\
& f_{y y}=\left(f_{\xi \xi} \xi_{y}+f_{\xi \eta} \eta_{y}\right) \xi_{y}+f_{\xi} \xi_{y y}+\left(f_{\eta \xi} \xi_{y}+f_{\eta \eta} \eta_{y}\right) \eta_{y}+f_{\eta} \eta_{y y} .
\end{aligned}
$$

Adding Eqs. (8a) and (8b), we find

$$
f_{x x}+f_{y y}=\left(f_{\xi \xi}+f_{\eta \eta}\right)\left(\xi_{x}^{2}+\xi_{y}^{2}\right) .
$$

If we have two such functions, $f$.and $g$, it follows from Eqs. $(7 a, b)$ that

$$
f_{x} g_{x}+f_{y} g_{y}=\left(f_{\xi} g_{\xi}+f_{\eta} g_{\eta}\right)\left(\xi_{x}{ }^{2}+\xi_{y}{ }^{2}\right)
$$

An infinitesimal area in the $\xi, \eta$ plane is related to one in the $x, y$ plane according to the rule

$$
d \xi d \eta=\frac{\partial(\xi, \eta)}{\partial(x, y)} d x d y=\left(\xi_{x}{ }^{2}+\xi_{y}{ }^{2}\right) d x d y
$$

A point source at position $x_{0}, y_{0}$ has the form

$$
S=Q \delta\left(x-x_{0}, y-y_{0}\right)
$$

where $Q$ has the dimensions cal $\mathrm{cm}^{-1} \mathrm{sec}^{-1}$, and $\delta$ is Dirac's delta function. Delta functions transform under change of coordinates according to

$$
\delta\left(x-x_{0}, y-y_{0}\right) d x d y=\delta\left(\xi-\xi_{0}, \eta-\eta_{0}\right) d \xi d \eta .
$$


which, in view of Eq. (11), can be written

$$
\delta\left(x-x_{0}, y-y_{0}\right)=\delta\left(\xi-\xi_{0}, \eta-\eta_{0}\right)\left(\xi_{x}{ }^{2}+\xi_{y}{ }^{2}\right)
$$

When the flow is incompressible and irrotational, $v$ can be written as the gradient of a potential $\phi$ :

$$
\begin{aligned}
& \mathbf{v}=\nabla \phi, \\
& \nabla^{2} \phi=0 .
\end{aligned}
$$

Using Eqs. (14) we can write Eq. (3) in two dimensions for a point source as

$$
k\left(T_{x x}+T_{y y}\right)-\rho c_{p}\left(\phi_{x} T_{x}+\phi_{y} T_{y}\right)+Q \delta\left(x-x_{0}, y-y_{0}\right)=0
$$

The equation for $\phi(14 h)$ is then

$$
\phi_{x x}+\phi_{y y}=0
$$

From Eqs. (9), (10), and (13b), we see that (15a) and (15b) have exactly the same form in terms of $\xi$ and $\eta$ that they have in terms of $x$ and $y$; that is,

$$
\begin{aligned}
& k\left(T_{\xi \xi}+T_{\eta \eta}\right)-\rho c_{p}\left(\phi_{\xi} T_{\xi}+\phi_{\eta} T_{\eta}\right)+Q \delta\left(\xi-\xi_{0}, \eta-\eta_{0}\right)=0, \\
& \phi_{\xi \xi}+\phi_{\eta \eta}=0 .
\end{aligned}
$$

Furthermore, the boundary conditions are likewise conformally invariant. The hydraulic boundary condition is that the velocity be parallel to boundary surfaces; the thermal boundary condition is that the isotherms be perpendicular to boundary' surfaces (which are taken impervious to heat). Sincé the anngle between two curves is preserved in conformal mapping, the boundary conditions are conformally invariant. Thus, if we have any solution to a problem of convective diffusion with potential flow, heat-impervious boundaries, and point sources, we get another solution of the same type by conformal transformation.

\section{POINT SOURCE IN A UNIFORMLY FLOWING, INFINITE MEDIUM}

We solve Eq. $(15 a)$ in an infinite medium. For convenience we locate the point source at the origin $\left(x_{0}\right.$ $=0, y_{0}=0$ ). Furthermore, we choose the $x$ axis to point in the direction of flow. Then (15a) becomes

$$
k\left(T_{x x}+T_{y y}\right)-\rho c_{p} v T_{x}+Q \delta(x, y)=0
$$

where $\mathrm{v}$ is the speed of the uniform flow. We try a solution of the form

$$
T=T_{\infty}+A \exp (\mathrm{v} x / 2 \alpha) R(\mathrm{v} r / \alpha)
$$

where 


$$
\begin{aligned}
& \alpha=k / \rho c_{p}=\text { thermal diffusivity }\left(\mathrm{cm}^{2} / \mathrm{sec}\right), \\
& r=\left(x^{2}+y^{2}\right)^{1 / 2},
\end{aligned}
$$

$T_{\infty}$ is the temperature of the flowing medium far from the source, and $A$ is a constant of integration. Substitution of Eq. (18a) intu (17) yields the differential equation for $R$ :

$$
\frac{d^{2} R}{d r^{2}}+\frac{1}{r} \frac{d R}{d r}-\left(\frac{\mathrm{v}}{2 \alpha}\right)^{2} R=0
$$

valid for $r>0$. Equation (19) is Bessel's equation of order zero when written for the variable $i v r / 2 \alpha$; hence the solution we seek is a zero-order Bessel function of imaginary argument. It must vanish when $r \rightarrow \infty$, and it must be singular at $r=0$. This identifies the solution as the zero-order Hankel function of the first kind:

$$
R=i \mathrm{H}_{0}{ }^{(1)}(i v r / 2 \alpha) \text {. }
$$

The factor $i$ outside has been added to make $R$ real.

The constant $A$ is determined by conditions near the source. For

$$
\mathbf{q}=A k \exp (\mathrm{v} x / 2 \alpha)(\mathrm{v} / 2 \alpha)\left[\mathrm{H}_{0}{ }^{(1)^{\prime}}(i r v / 2 \alpha) \mathrm{e}_{r}+i \mathrm{H}_{0}{ }^{(1)}(i r v / 2 \alpha) \mathrm{e}_{x}\right]+\rho c_{p} \mathrm{v} T_{\infty} \mathrm{e}_{x},
$$

where $\mathrm{e}_{x}$ and $\mathrm{e}_{r}$ are unit vectors in the $x$ and $r$ directions, respectively. Then

$$
Q=\lim _{r \rightarrow 0}\left(r \int_{0}^{2 \pi} \mathrm{q} \cdot \mathrm{e}_{r} d \beta\right)=4 A k
$$

where $\beta=\arg (\mathbf{r})$ is the polar angle of $r$. Here we have used the relations

$$
\begin{aligned}
& i \mathrm{H}_{0}{ }^{(1)}(i y) \approx \frac{2}{\pi} \cdot \ln \left(\frac{2}{\gamma y}\right), \quad y \ll 1, \\
& \mathrm{H}_{0}{ }^{(1)^{\prime}}(i y)=-\mathrm{H}_{1}{ }^{(1)}(i y) \approx 2 / \pi y, \quad y \ll 1,
\end{aligned}
$$

where $\gamma$ is the exponential of Euler's constant. Finally, then

$$
T-T_{\infty}=\frac{Q}{4 k} \exp \left(\frac{v x}{2 \alpha}\right) i \mathrm{H}_{0}(1)\left(\begin{array}{c}
i v r \\
2 \alpha
\end{array}\right)
$$

Equation (24) gives the temperature distribution due to a point source of strength $Q$ at the origin in a uniformly flowing infinite medium.

Typical values of $\alpha$ are of the order of magnitude of $10 \mathrm{ft}^{2} / \mathrm{sec}$, and typical values of $\mathrm{v}$ are of the order of $1 \mathrm{ft} / \mathrm{sec}$. The characteristic length $2 \alpha / \mathrm{v}$ is then of the order of $20 \mathrm{ft}$. Far-field calculations generally apply at distances much greater than $20 \mathrm{ft}$, so that $\mathrm{vr} / 2 \alpha$ is generally $\gg 1$. Now

$$
i \mathrm{H}_{0}^{(1)}(i y) \cdots(2 / \pi y)^{1 / 2} e^{-y}, \quad y \geqslant 1
$$


so that

$$
T \cdots T_{\infty}^{i} \sim \frac{Q}{4 k}(4 \alpha / \pi v r)^{1 / 2} \exp [-v(r-x) / 2 \alpha]
$$

When $v r / 2 \alpha \gg 1, v(r-x) / 2 \alpha$ is only small when $r$ and $x$ are nearly the same. Now $r-x=y^{2} / 2 x$ to lowest order in $y$. with increasing accuracy for fixed $y^{2} / 2 x$ as $x$ increases. Hence, to lowest order in $y$,

$$
T-T_{\infty}=\frac{Q}{4 k}(4 \alpha / \pi v x)^{1 / 2} \exp \left(-v y^{2} / 4 \alpha x\right)
$$

This is the result quoted by Edinger and Polk ${ }^{2}$ when longitudinal diffusion is neglected [i.e., when the term $T_{x x}$ is dropped in (15a)]. It is only valid far from the source, but this suffices for practical purposes.

\section{USE OF THE VELOCITY POTENTIAL AND THE STREAM FUNCTION AS COORDINATES}

Let us now consider a source $Q \delta\left(x-x_{0}, y-y_{0}\right)$ in an arbitrary prescribed potential tlow field. The transformation from $x, y$ to $\phi(x, y), \psi(x, y)$, where $\phi$ is the (known) velocity potential and $\psi$ is the (known) stream function of the tlow, is conformal. Hence we can choose $\psi$ and $\psi$ to be $\xi$ and $\eta$ respectively. Equation (16a) then becomes

$$
k\left(T_{\phi \phi}+T_{\psi \psi}\right)-\rho c_{p} T_{\phi}+Q \delta\left(\phi-\phi_{0}, \psi-\psi_{0}\right)=0
$$

Equation (25) has the same form as Eq. (17) when $v=1$. We know the solution to the latter equation in an infinite medium [Eq. (24)], but this sulution does not immediately apply to practical problems for the following reason. In practical flow problems the flow is usually bounded by one or more solid boundaries; in fact, it is often the presence of these solid boundaries that causes the flow field to be nonunitorm. Un these solid boundaries the stream function has constant values. If the flow is confined to a simply connected region between two such boundaries, then in the $\phi, \psi$ plane the flow is confincd to a strip parallel to the $\phi$ axis. It is within this strip that we must solve Eq. (25).

The isotherms are always perpendicular to solid boundaries, the latter being.taken impervious to heat. Since angles are preserved by conformal mapping, the isotherms are also perpendicular to the sides of the strip in the $\phi, \psi$ plane. Let $\psi_{1}<\psi_{2}$ be the values of the stream function on the solid boundaries. Then the boundary conditions on $T$ at $\psi=\psi_{1}$ and $\psi=\psi_{2}$ are

$$
T_{\psi}=n \text { at } \psi=\psi_{1} \text { and } \psi=\psi_{2} \text {. }
$$

We can satisfy the conditions in Eq. (26) if we put image sources at the points

$$
\begin{aligned}
& \phi=\phi_{0} \\
& \psi=2 \psi_{1}-\psi_{0}, \psi_{0} \pm 2 n\left(\psi_{2}-\psi_{1}\right), 2 \psi_{1}-\psi_{0} \pm 2 n\left(\psi_{2}-\psi_{1}\right),
\end{aligned}
$$

where $n=1,2,3, \ldots$. To prove that these images suffice we must show that the system of image sources together with the real source lie symmetrically around the lines $\psi=\psi_{1}$ and $\psi=\psi_{2}$. To do this it is enough 
to break up the system of sources into pairs, each of whose $\psi$ values add up to $2 \psi_{1}$ or $2 \psi_{2}$. The sets of pairs that add up to $2 \psi_{1}$ are $\psi_{0}, 2 \psi_{1}-\psi_{0}$ and $\psi_{0}+2 n\left(\psi_{2}-\psi_{1}\right), 2 \psi_{1}-\psi_{0}-2 n\left(\psi_{2}-\psi_{1}\right)$, where $n=$ $\pm 1, \pm 2, \ldots$. The sets of pairs that add up to $2 \psi_{2}$ are $\psi_{0}+2 n\left(\psi_{2}-\psi_{1}\right), 2 \psi_{1}-\psi_{0}-(2 n-2)\left(\psi_{2}-\psi_{1}\right)$, where $n=0, \pm 1, \pm 2, \ldots$. This means that the solution of Eqs. (25) and (26) we seek in the strip $\psi_{1}<\psi_{2}<\psi_{2}$ is the sum of the infinite medium solutions (24) for its real source and all its image sources; that is,

$T-T_{\infty}=\sum_{n=-\infty}^{+\infty}\left\{F\left[\phi-\phi_{0}, \psi-\left(\psi_{0}+2 n\left\{\psi_{2}-\psi_{1}\right\}\right)\right]+F\left[\phi-\phi_{0}, \psi-\left(2 \psi_{1}-\psi_{0}+2 n\left\{\psi_{2}-\psi_{1}\right\}\right)\right]\right\}$

where $F(x, y)$ is the function of $x$ and $y$ defined by the right-hand side of Eq. (24) when $v=1$.

To facilitate rapid evaluation of temperature rises from Eq. (28), a computer program has been written in the BASIC. language for the PDP-10 time-share system at ORNL. The program is named STRIP, and it calculates the quantity $\theta=4 k\left(T-T_{\infty}\right) / Q$ at any point in an arbitrary two-dimensional potential flow field. The program is written in the conversational mode, and an order to RUN will begin a conversation with the computer in which the user is instructed how to proceed.

The program requests the user to supply the following information:

1. Two functions of position specifying, respectively, the velocity potential and the stream function of the flow.

2. The abscissa and ordinate of a point on each boundary. Since the boundaries must be streamlines (on which $\psi=$ constant), one point on each boundary is all that is needed to calculate $\psi_{1}$ and $\psi_{2}$.

3. The eddy diffusivity.

4. The position of the source. Either a point source or a line source can be handled. The line source is specified by giving the position of its end points and is actually handled by the program as an array of $N 1$ equally spaced point sources each, of strength $Q / N 1$. (Presently $N 1=100$, but any value can be used.) Should a weighting factor be desired along the line source, one can be introduced by a comparatively single reprogramming of STRIP.

When the above information has been supplied, the program responds with a query as to whether the user wants the value of $\theta$ at a field point. If the answer is yes, the program responds with a request for the abscissa and ordinate of the field point, and upon receipt of these data responds with the value of $\theta$. The user is then queried again as to whether he wants the value of $\theta$ at a field point. If the answer is no, the program stops, but a message is presented saying a new case may be run by giving the order RUN.

The program will print an error message if either the source or the field point does not lie between the boundaries.

The location of the origin of coordinates is arbitrary.

Owing to the complexity of formula (28), it cannot be inverted easily, and isotherms cannot be calculated directly. The best way to calculate isotherms is to use STRIP to obtain temperature rises at a lattice of points and then graphically interpolate for the desired temperature rise. Figure 1 shows isotherms obtained in this way for the flow in a $5^{\circ}$ converging wedge. Figure 2 shows isotherms for the flow around a half cylinder. A listing of the program and a typical conversation with the computer are given in the Appendix. 


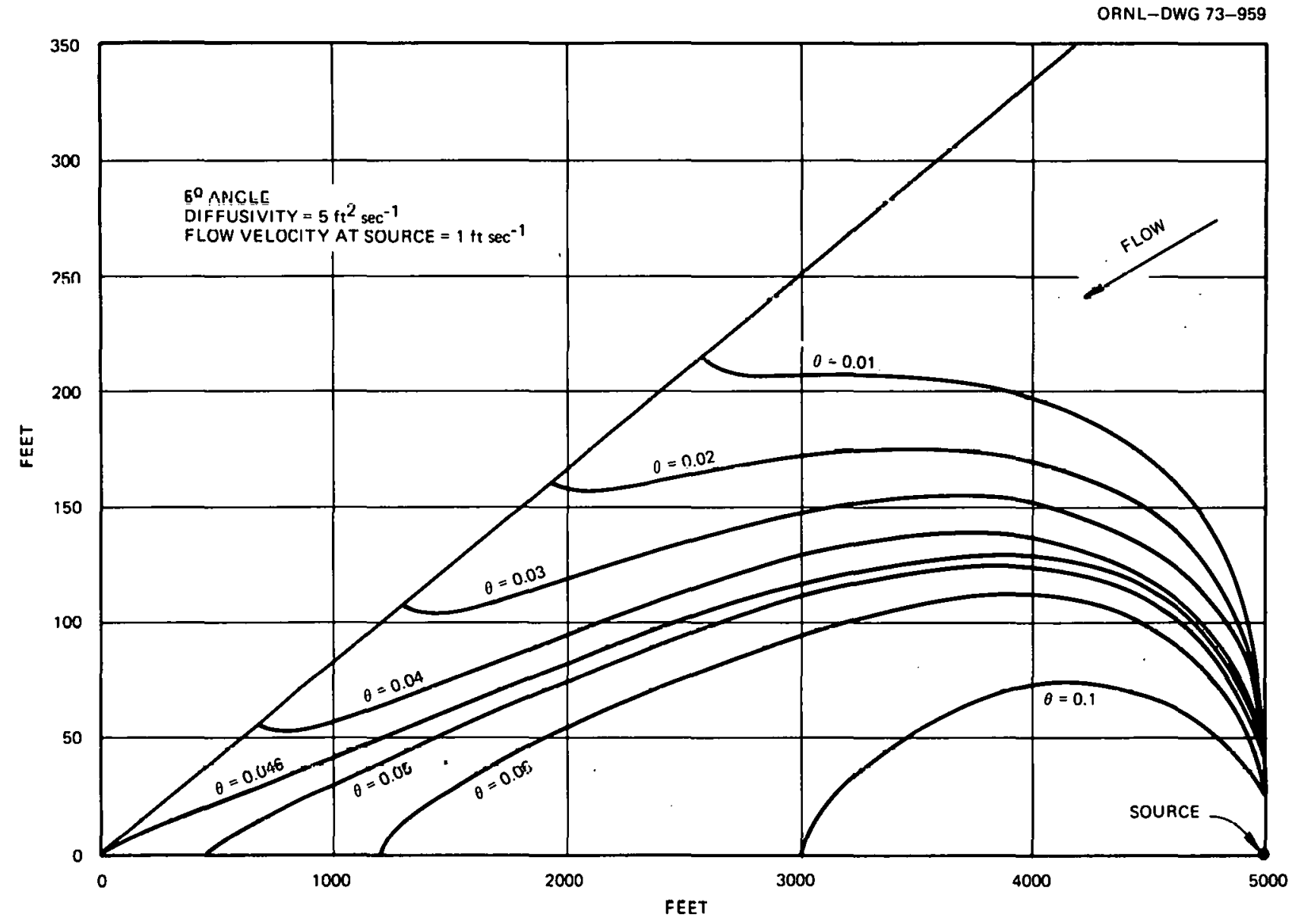

Fig. 1 . Isotherms in the converging flow in a $5^{\circ}$ wedge. 


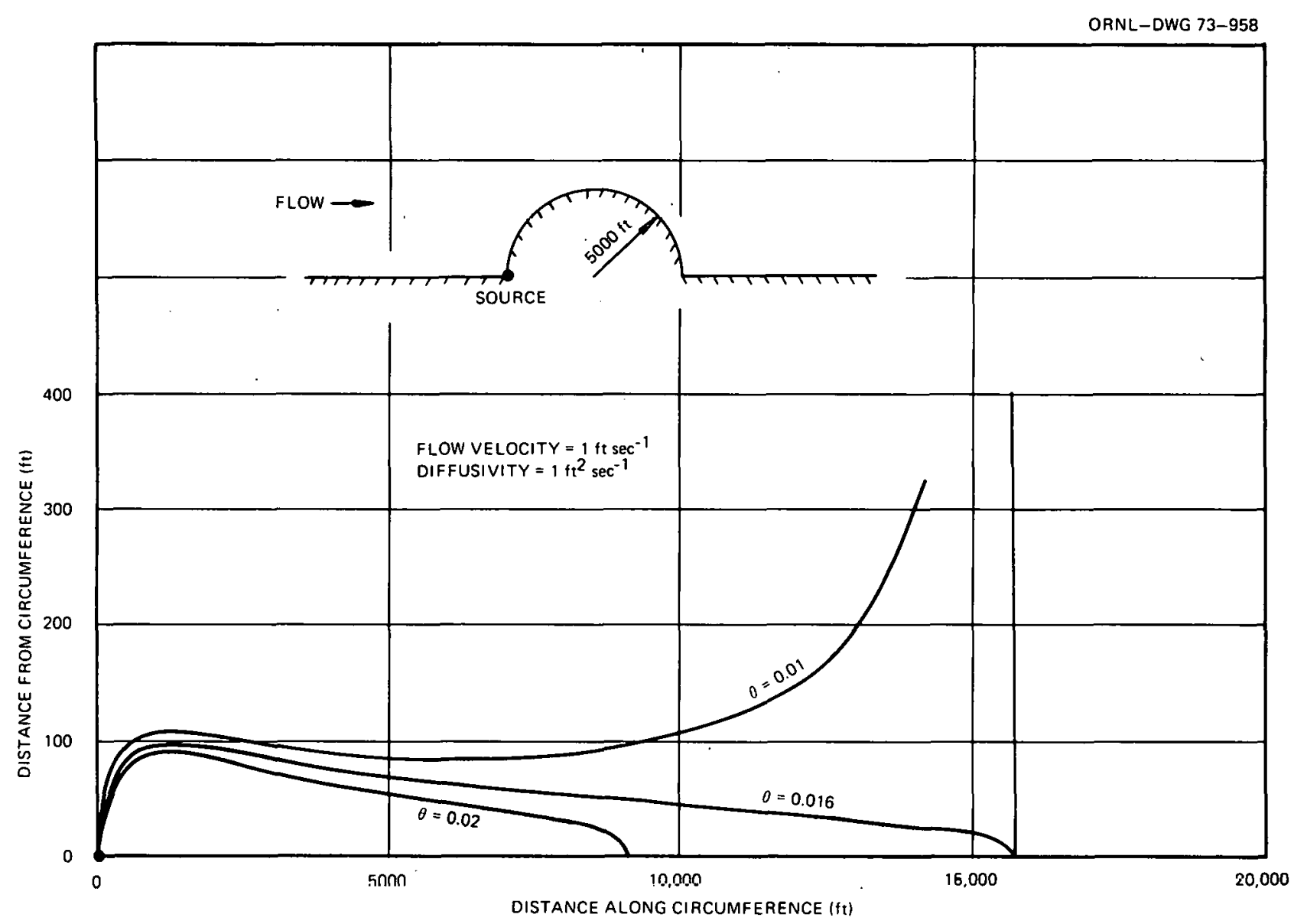

Fig. 2. Isotherms in the flow around a half cylinder. 


\section{SURFACE COOLING}

Surface heat exchange can be expressed in terms of an exchange coefficient $K\left(\mathrm{cal} \mathrm{cm}^{-2} \mathrm{sec}^{-1} \mathrm{deg}^{-1}\right)$ in the following way according to Harleman and Stolzenbach: ${ }^{1}$

$$
q=K\left(T-T_{\infty}\right),
$$

where $q$ is the heat flux out of the surface of the body of water, and $T$ is the surface temperature. If we assume complete vertical mixing in the body of water we are studying (in order to keep the problem two dimensional), the heat loss (29) appears as a distributed heat sink with a local strength $(K / d)\left(T-T_{\infty}\right)$, where $d$ is the (uniform) depth; $(K / d)\left(T-T_{\infty}\right.$ ) has the dimensions $\mathrm{cal} \mathrm{cm}^{-3} \mathrm{sec}^{-1}$ as a source or sink should. With this sink term, Eq. (3) for a point source can be written

$$
-k \nabla^{2}(\delta T)+\rho c_{p} v \cdot \nabla(\delta T)=Q \delta\left(x-x_{0}, y-y_{0}\right)-(K / d)(\delta T),
$$

where $\delta T=T-T_{\infty}$

In a uniformly flowing, infinite medium Eq. (30) can also be solved by a substitution of the form (18a). Now the function $R$ satisfies the equation

$$
\frac{d^{2} R}{d r^{2}}+\frac{1}{r} \frac{d R}{d r}-\left(\frac{v}{2 \alpha}\right)^{2}\left(1+\frac{4 K \alpha}{\rho c_{p} d v^{2}}\right) R=0
$$

We again identify $R$ with the zero-order Hankel function of the first kind, so that

$$
T=T_{\infty}+\frac{Q}{4 k} \exp \left(\frac{v x}{2 \alpha}\right) i \mathrm{H}_{0}{ }^{(1)}\left[\frac{i v r}{2 \alpha}\left(1+\frac{4 K \alpha}{\rho c_{p} d v^{2}}\right)^{1 / 2}\right]
$$

the constant $A$ having again been determined by a calculation similar to that following Eq. (21).

According to Harleman and Stolzenbach, ${ }^{1}$ typical values for $K$ range from 100 to $200 \mathrm{Btu} \mathrm{ft}^{-2} \mathrm{day}^{-1}$ $\left({ }^{\circ} \mathrm{F}\right)^{-1}$. Choosing $K=150 \mathrm{Btu} \mathrm{ft}^{-2} \mathrm{day}^{-1}\left({ }^{\circ} \mathrm{F}\right)^{-1}, \alpha=10 \mathrm{ft}^{2} / \mathrm{sec}$, and $\mathrm{v}=1 \mathrm{ft} / \mathrm{sec}$, we find

$$
\frac{4 K \alpha}{\rho n p^{2} d v^{2}}=4.45 \times 10^{-5}
$$

for a typical depth $d$ of $25 \mathrm{ft}$. When $v r / 2 \alpha \gg 1$, Eq. (32) becomes

$$
T-T_{\dot{\infty}}+\frac{Q}{4 k}(4 \mathrm{kv} / \pi v)^{1 / 2} \exp _{\mathrm{p}}[-\mathrm{v}(r-x) / 2 . r]\left(1+\frac{4 K \alpha}{\rho c_{p} \mathrm{v}^{2} d}\right)^{-1 / 4} \operatorname{cxp}\left(\begin{array}{c}
K r \\
\rho c_{p} d \mathrm{v}
\end{array}\right)
$$

by again using (23c). The last two factors in Eq. (34) represent corrections to (24a) to account for surface cooling. The first correction is very close to 1 , and for practical purposes it can be ignored. The second factor is less than a $10 \%$ correction when $r \leqslant \rho c_{p} d v / 10 K=9.00 \times 10^{4} \mathrm{ft}=17$ miles. At this distance, the center-line temperature rise is $0.00436(Q / 4 k)$, and the half-width of the plume $(4 \alpha x / \mathrm{v})^{1 / 2}=1900 \mathrm{ft}$. If the total rate of heat release is $1000 \mathrm{MW}, Q / 4 k=15.2^{\circ} \mathrm{F}$ with the values of $\alpha$ and $d$ we have been using. The center-line temperature rise is then $0.0661^{\circ} \mathrm{F}$. These estimates make it clear that surface cooling may sometimes be neglected in the calculation of isotherms of practical importance. 


\section{JOINING THE FAR-FIELD SOLUTION TO THE NEAR-FIELD SOLUTION}

The near field is often created by a single jet or by a row of jets. The row of jets produces a distribution of excess temperature that is uniform over a line, and the far field produced by a line source can be directly calculated using STRIP. When the near field is created by a single jet, the distribution of excess temperature can of ten be described with very good accuracy by a Gaussian function. Such a Gaussian source may be used in STRIP by using a line source with Gaussian weights. This involves a rather simple reprogramming of STRIP as mentioned before. However, even this reprogramming can be avoided under certain circumstances, described below, by replacing the Gaussian temperature distribution by a virtual point source suitably located.

Let us first consider a uniform flow field flowing in the $x$ direction. If the heat source $S$ is distributed, then

$$
\delta T(x, y)=\int \delta T_{0}\left(x-x^{\prime}, y-y^{\prime}\right) \frac{S\left(x^{\prime}, y^{\prime}\right)}{Q} d x^{\prime} d y^{\prime}
$$

where $\delta T_{0}$ is the function on the right-hand side of (24). Now

$$
\frac{S(x, y)}{Q}=\frac{\exp \left(-y^{2} / b^{2}\right)}{b \sqrt{\pi}} \delta(x)
$$

represents a Gaussian source distributed in the $y$ direction (across the flow) with a half-width at $1 / e X$ maximum of $b$ and centered on the origin of coordinates. If we replace $\delta T_{0}$ by its asymptotic form (24b), then (34) becomes the same as (24b) except that $x$ is replaced by $x+b^{2} v / 4 \alpha$. For the right-hand side of (35) is then proportional to the convolution of two normalized Gaussians, which by DeMoivre's theorem is again a normalized Gaussian whose half-width is the square root of the sum of the squares of the half-widths of the two convoluted Gaussians. Thus the far field can be obtained by placing a virtual point source of total strength $Q$ a distance $b^{2} \mathrm{v} / 4 \alpha$ upstream of the center of the Gaussian temperature distribution created by the jet. The same trick can be used in a nonuniform flow field as long as the flow velocity is relatively constant for a distance $\sim b^{2} v / 4 \alpha$ upstream of the source. Typical values of $b$ are of the order of $25 \mathrm{ft}$, so that $b^{2} \mathrm{v} / 4 \alpha=16 \mathrm{ft}$ for the figures we have been using. The assumption of the constancy of the flow velocity over such a short distance is of ten quite good. The use of $(24 b)$ implies that the nearest boundary is at least several units of $b$ from the virtual source.

\section{HALF-SPACE WITH A BREAKWATER}

The Schwarz-Christoffel transformation

$$
(z-a)^{2}=\left[\zeta-\left(a^{2}+c^{2}\right)^{1 / 2}\right]^{2}-c^{2},
$$

where $a$ and $c$ are real numbers, maps the upper half-plane in the $\zeta$ plane $(\zeta=\xi+i \eta)$ onto the upper half-plane in the $z$ plane $(z=x+i y)$ with an obstacle (breakwater) of length $c$ occurring in the $z$ plane at $x$ $=a$ (cf., e.g., Fig. 3). Let the $x$ axis be the shore, and let the offshore flow be parallel to the $x$ axis for $y \gg c$. We can calculate the temperature at a point $(x, y)$ due to a line source of strength $Q$ at $\left(x_{0}, y_{0}\right)$ as follows: Using the transformation (37) we find the coordinates of the image points $(\xi, \eta)$ and $\left(\xi_{0}, \eta_{0}\right)$ of $(x, y)$ and $\left(x_{0}, y_{0}\right)$ respectively. In the $\zeta$ plane, the tlow is uniform and parallel to the $\xi$ axis everywhere in the upper 


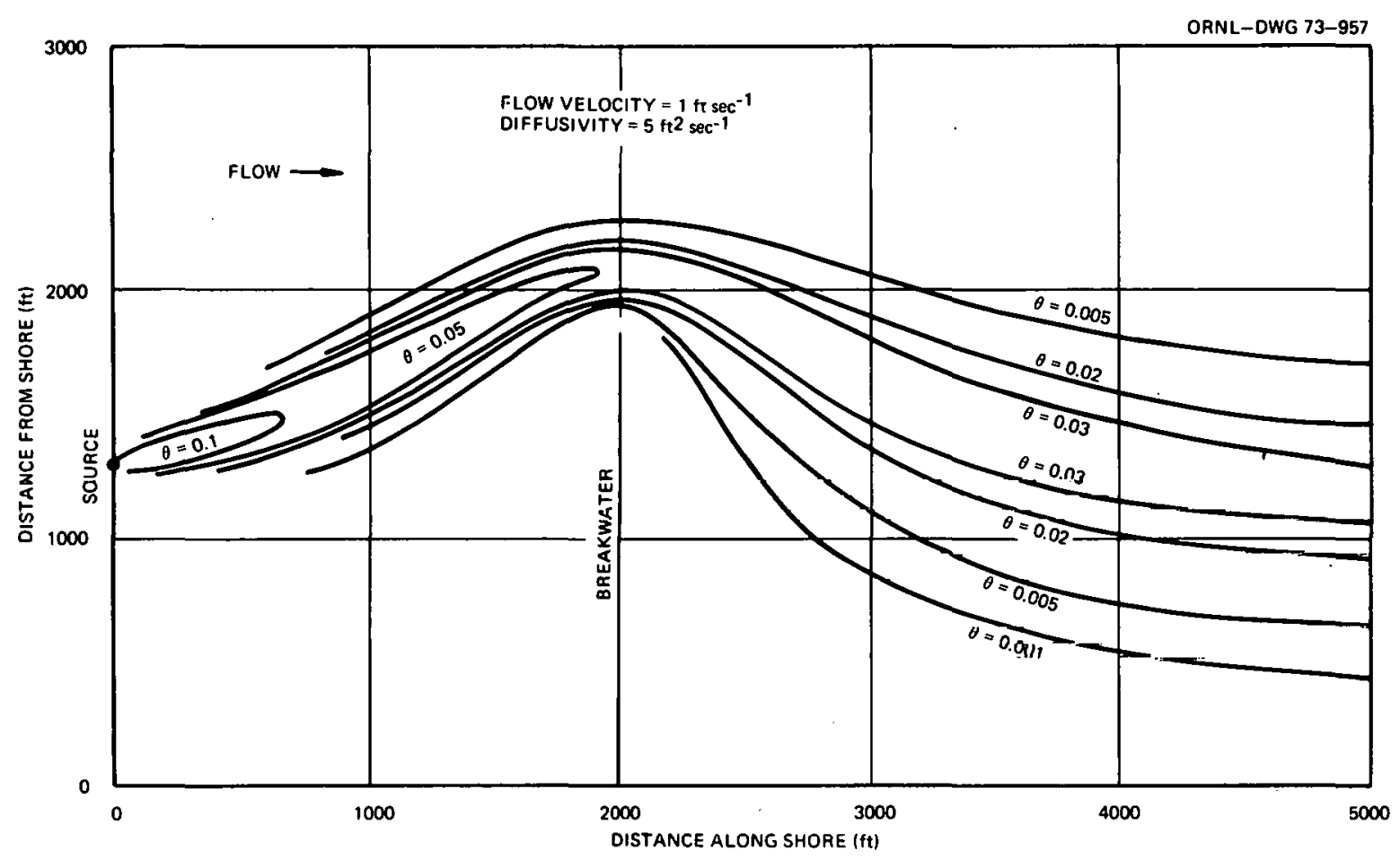

Fig. 3. Isotherms in the flow field around a breakwater.

half-plane. The $\xi$ axis, being the image of the $x$ axis (shore) together with the breakwater, is perpendicular to the isotherms in the $\zeta$ plane and therefore represents the shore there. If we place an image source of strength $Q$ in the $\zeta$ plane at $\left(\xi_{0},-\eta_{0}\right)$, the temperature rise we seek is the sum of those produced individually at $(\xi, \eta)$ by sources at $\left(\xi_{0}, \eta_{0}\right)$ and $\left(\xi_{0},-\eta_{0}\right)$, the individual contributions being calculated with Eq. (24).

A second BASIC program named FARFLD has been written to facilitate calculations based on the above ideas. Whereas SIRIP calculates a dimensionless temperature rise and requires no source strength as input, FARFLD calculates temperature rises in degrees Fahrenheit and requires data on the source as input.

The command RUN causes FARFLD to ask for the following quantities:

1. the volume flow rate through the condensor, $\mathrm{ft}^{3} / \mathrm{sec}$;

2. the temperature rise in the condenser, ${ }^{\circ} \mathrm{F}$;

3. the eddy diffusivity, $\mathrm{ft}^{2} / \mathrm{sec}$;

4. the offshore flow velocity, $\mathrm{ft} / \mathrm{sec}$;

5. the depth, $\mathrm{ft}$;

6. the position of the foot of the breakwater and the length of the breakwater (if one is present), $\mathrm{ft}$.

Either a point or a line source is allowed. If a point source is elected, FARFLD asks for its coordinates in feet; if a line source is elected, FARFLD asks for the coordinates of its end points.

When the above information has been supplied, the program responds with a query as to whether the user wants the temperature rise at a field point. If the answer is yes, the program responds with a request for the coordinates of the field point and, upon receipt of these data, responds with the temperature rise in 
degrees Fahrenheit. The user is then queried again as to whether he wants the temperature rise at a field point. If the answer is no, the program stops with a message saying a new case may be run by giving the order RUN.

Again isotherms are best calculated by the procedure previously outlined in connection with STRIP.

Figure 3 shows the results of a typical calculation done using FARFLD. A listing of the program as well as a typical conversation with the computer may be found in the Appendix.

\section{ADDITIONAL REMARKS}

Sometimes the longitudinal and lateral eddy diffusivities are not the same. As we saw from the discussion following Eq. (24), longitudinal diffusion is not important except close to the source (where a far-field calculation does not apply anyway). Little error is then incurred if we replace the true value of the longitudinal eddy diffusivity by the value of the lateral eddy diffusivity. When this replacement is made, the methods of this report can be employed. Thus, when the longitudinal and lateral diffusivities are different, $k$ should have the value of the lateral diffusivity.

The results in this report can be used not only for the diffusion of heat, but also for the diffusion of dissolved substances. Any equation in this report can be transcribed for the diffusion of dissolved substances if the unit of heat (calorie) is replaced in the definition of $S$ and $Q$ by a unit of amount, for example, mole, and if the quantity $\rho c_{p} \delta T$ is replaced by the concentration of the dissolved substance in the corresponding units, for example, moles $/ \mathrm{cm}^{3}$ when the unit of amount is mole. There is no surface exchange of nonvolatile dissolved substances; but if the dissolved substance is radioactive, a distributed sink exists with a strength proportional to the concentration. The surface heat loss (29) was also treated as a distributed sink, and the formulas of the section of surface cooling can be rewritten for a dissolved radioactive substance by making the changes noted above and by replacing $\rho c_{p} d / K$ by the mean life of the decay. When, typically, $K=150 \mathrm{Btu} \mathrm{ft}^{-2} \mathrm{day}^{-1}\left({ }^{\circ} \mathrm{F}\right)^{-1}$ and $d=25 \mathrm{ft}$, the mean life of the equivalent decay is about ten days. This means that radioactive decay has much less effect on the diffusion of a dissolved substance than surface cooling has on the diffusion of heat when the mean life of the decay is long compared with ten days. If no distinction is made between radioactive parent and daughter, there is no "surface cooling." 


\section{Symbols}

a Number in the conformal mapping given in Eq. (37)

$b \quad$ Half-width of Gaussian source in Eq. (36)

c Number in the conformal mapping given in Eq. (37)

$c_{p} \quad$ Heat capacity (cal g $\mathrm{g}^{-1} \mathrm{deg}^{-1}$ )

d Depth $(\mathrm{cm})$

$f, g$ Any functions of $x, y$

$F \quad$ Function of $x, y$ defined by the right-hand side of Eq. (24) when $v=1$

$k \quad$ Thermal (eddy) conductivity (cal cm $\mathrm{cm}^{-1} \mathrm{sec}^{-1} \mathrm{deg}^{-1}$ )

$K \quad$ Surface heat exchange coefficient $\left(\mathrm{cal} \mathrm{cm} \mathrm{cec}^{-1} \mathrm{deg}^{-1}\right)$

q Heat flux vector (cal cm-2 $\left.\mathrm{sec}^{-1}\right)$

$Q \quad$ Point source strength (cal cm$\left.{ }^{-1} \mathrm{sec}^{-1}\right)$

r Radius vector (cm)

$R \quad$ Function defined in Eq. (18a)

$S \quad$ Heat source density (cal $\mathrm{cm}^{-3} \mathrm{sec}^{-1}$ )

$T \quad$ Temperature (deg)

$\delta T \quad T-T_{\infty}$

$v \quad$ Flow velocity vector $(\mathrm{cm} / \mathrm{sec})$

$x, y, z$ Space coordinates $(\mathrm{cm})$

$\alpha \quad$ Eddy diffusivity $=k / \rho c_{p}\left(\mathrm{~cm}^{2} / \mathrm{sec}\right)$

$\beta$ Polar angle of $r$

$\gamma \quad$ Exponential of Euler's constant $=1.781$

$\delta \quad$ Dirac's delta function

$\zeta \quad$ Image of $z$ in the conformal mapping of Eq. (37)

$\theta \quad 4 k\left(T-T_{m}\right) / O$

$\xi, \eta \quad$ Space coordinates after conformal transformation $(\mathrm{cm})$

$\rho \quad$ Density $\left(\mathrm{g} / \mathrm{cm}^{3}\right)$

$\phi \quad$ Velocity potential $\left(\mathrm{cm}^{2} / \mathrm{sec}\right)$

$\psi \quad$ Stream function $\left(\mathrm{cm}^{2} / \mathrm{sec}\right)$

\section{Subscripts}

$0 \quad$ Position of point source

$\infty \quad$ Ambient temperature

$x, y, \xi, \eta, \phi, \psi \quad$ Partial differentiation

1,2

Values of stream function on boundaries 
APPENDIX 


\section{STRIP}

10 PRINT "IISFR: TYPE "LISTNH 1OB-350" FDR MESSAGE. AFTER MESSAGE"

20 PRINT "SUPPLY FUNCTIONS FNF(X,Y) AND FNP $(X, Y)$ - AND TYPE"

30 PRINT " "DELETE 10-40'. THEN TYPE 'RUN'."

40 STOP

50 REM **************************************************************

$100^{\circ}$ THIS PROGRAM CALCULATES THETA AT ANY PDINT I!N AN ARBITRARY T'NO-

$110^{\circ}$ DIMENSI ONAL POTENTIAL FLOW FIELD. FOR HEAT DIFFIJSION, THETA = $120^{\circ} 4 * K * T / S$, WHERE $K=$ EDDY THERMAL CONDUCTIVITY, $T=$ TEMPERATURE $130^{\circ}$ RISE ABOVE AMBIENT, AND $S=$ TOTAL SOURCE STRENGTH PER UNIT $140^{\circ}$ DEPTH. THE DIMENSIONS OF THFSE QUANTITIES ARE AS FOLLOWS: $150^{\circ}[K]=Q L \uparrow-1$ T $T-1$ DEGT-1, [T] $=D E G, A N D[S]=Q L \uparrow-1 T \uparrow-1$ $160^{\circ}$ (HERE Q REPRESENTS HEAT): ANY LINITS OF LENGTH AND TIME MAY BE $170^{\circ}$ USED, BUT THEY MUST BE USED CONSISTENTLY. FOR EXAMPI.E, IF $180^{\circ}$ LENGTHS ARE IN FEET AND TIME IS IN SECONDS, VELOCITIES MUST $190^{\circ} B E$ GI VEN IN FEET PER SECOND AND DIFFUSIVITIES IN FEET SQIJARED 200 $P E R$ SIECOND, ETC. ANY UNITS OF HEAT AND TEMPERAIURE MAY $210^{\circ} \mathrm{BE}$. USFD, BUT THEY MUST ALSO BE USED CONSISTENTLY. 220. FOR THE DIFFUSION OF DISSOLVED SUBSTANCES, THETA $=4 * A * C / S$, 230 WHEKE $A=$ EDDY DIFFUSIVITY, $C=$ CONCENTRATION, AND $S=$ IDTAL $240^{\circ}$ SOURCE STRENGTH PER UNIT DEPTH. THE DIUENSIONS OF THESE.

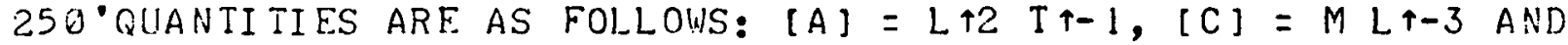
$260^{\circ}[S]=M L \uparrow-1$ T $\uparrow-1$ (HERE M REPRESENTS AMOUNT). 270. AGAI!, ANY UNI TS OF LENGTH AND TIME MAY BE USED AS LONG $280^{\circ}$ AS THEY ARE USED CONSISTENTLY. ANY UNITSS MAY BE USED FOR 29Ø AMOIJNT, E.G., MOLES, CURIES, GRAMS, ETC.

$300^{\circ}$ THE SOURCE CAN BE EI THER A POINT OR A LINF.

$310^{\circ}$ THE USER MISST SUPPLY TWO FUNCTIDNS, FNF(X,Y) AND FNP(X,Y), $320^{\circ}$ WHICH GIVE THE VELOCITY POTENTIAL AND STREAM FUNCTION, RESPEC$330^{\circ}$ TIVELY; AT THE POINT $(X, Y)$. THESE FUNCTIONS GO IN LINES $340^{\circ} 1008-1999$, BUT THE DEFINING LINES MUST USE LINES 1000 AND 1910. 350 NDTE CIIOICE OF SIGN: VELOCITY $\because+$ GIAD FINR $(X, Y)$.

36 REM $* * * * * * * * * * * * * * * * * * * * * * * * * * * * * * * * * * * * * * * * * * * * * * * * * * * * * * * * * * * * * * * *$ 1230 DEF $F$ NF $(X, Y)=X$

1010 DEF FNP $(X, Y)=Y$

2000 DEF FNB(P) $=.63662 * \operatorname{LOG}(1.1229 / R) *(1+1.2438 * R \uparrow 3.08)$

2010 DEF FNA $(R, X)$

2QDD IF R/2 > $=.75$ THEN 2050

2030 FNA $=\operatorname{EXP}(X / 2) * F N B(R / 2)$

2040 GO TO 2490

2052 IF $\left(R-Y_{1}\right) / 2>20$ THEN 2080

$2060 \mathrm{FNA}=.7979, * \operatorname{EXP}((X-R) / 2) /(\operatorname{SQR}(R / 2) *(1+.0952 *(R / 2) \uparrow(-.885)))$

$2070 \quad 60$ TO 2690

$208 \bar{B} \quad F N A=0$

2000 FNEND

$21010 \mathrm{REM} * * * * * * * * * * * * * * * * * * * * * * * * * * * * * * * * * * * * * * * * * * * * * * * * * * * * * * * * *$

2110 REM ****** INITIALIZE. SETS P2, P1, A1. TEMPDRARIES USED

2120 RTM ***** ARE X5, Y5, X6, Y6, T5, I6, Z1\$.

2130 PRINT "ABSCISSA AND ORIINATE OF A POINT ON ONE ROUNDARY";

2140 INPUT X5, Y5

2150 PRINT "ABSCISSA AND DRDIVATE. OF A POINT ON OTHER BDUNDARY";

2160 INPUT $X 6, Y 6$ 


\section{STRIP}

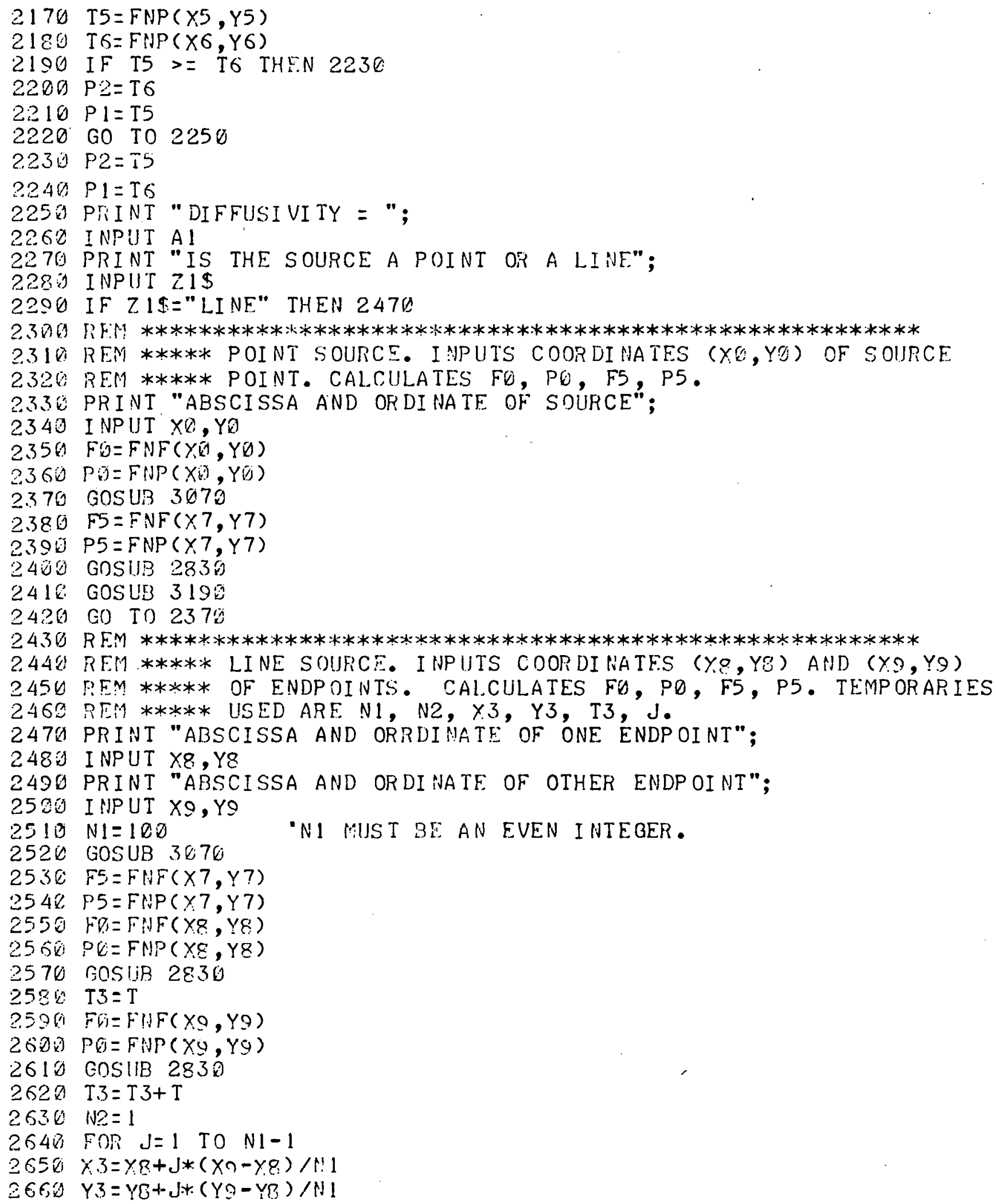




\section{STRIP}

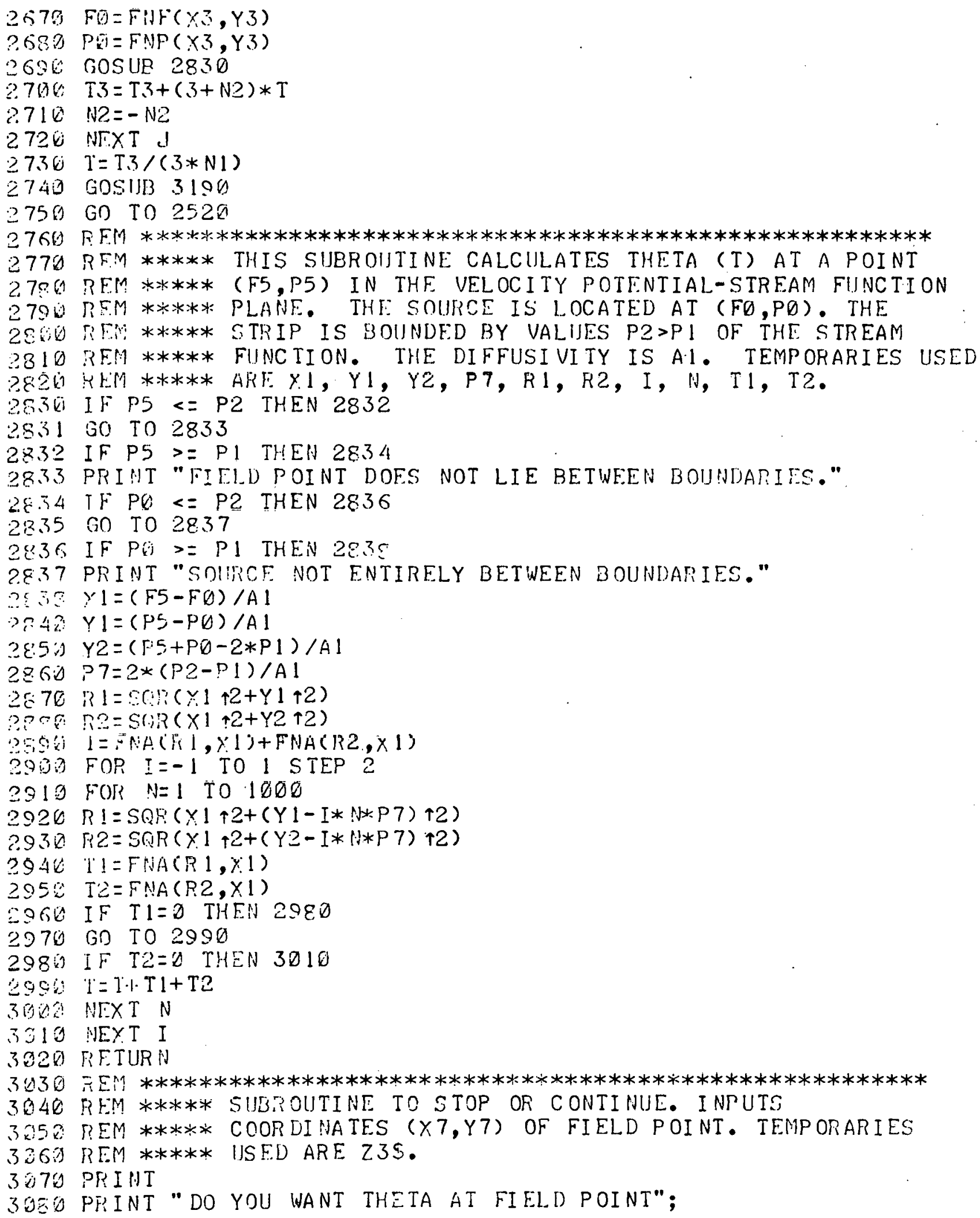




\section{STRIP}

3290 INPUT 235

3100 IF $239="$ YES" THEN 3140

3110 PRINT

3120 PRINT "TO RUN ANOTHER CASE TYPE "RUN"*

3130 STOP

3140 PRINT "ABSCISSA AND ORDINATE OF FIELD POINT";

3150 INPUT $\times 7, Y 7$

3166 RETURN

3170 ก IM $* * * * * * * * * * * * * * * * * * * * * * * * * * * * * * * * * * * * * * * * * * * * * * * * * * * * * * * * *$

3IEO REM ****** OUTPUT SUBR OUTINF.

3190 PRINT "THETA $=$ "T

3200 P.ETURN

$3216 \mathrm{P} E \mathrm{I}$; ************************************************************

3230 END 
FARFLD

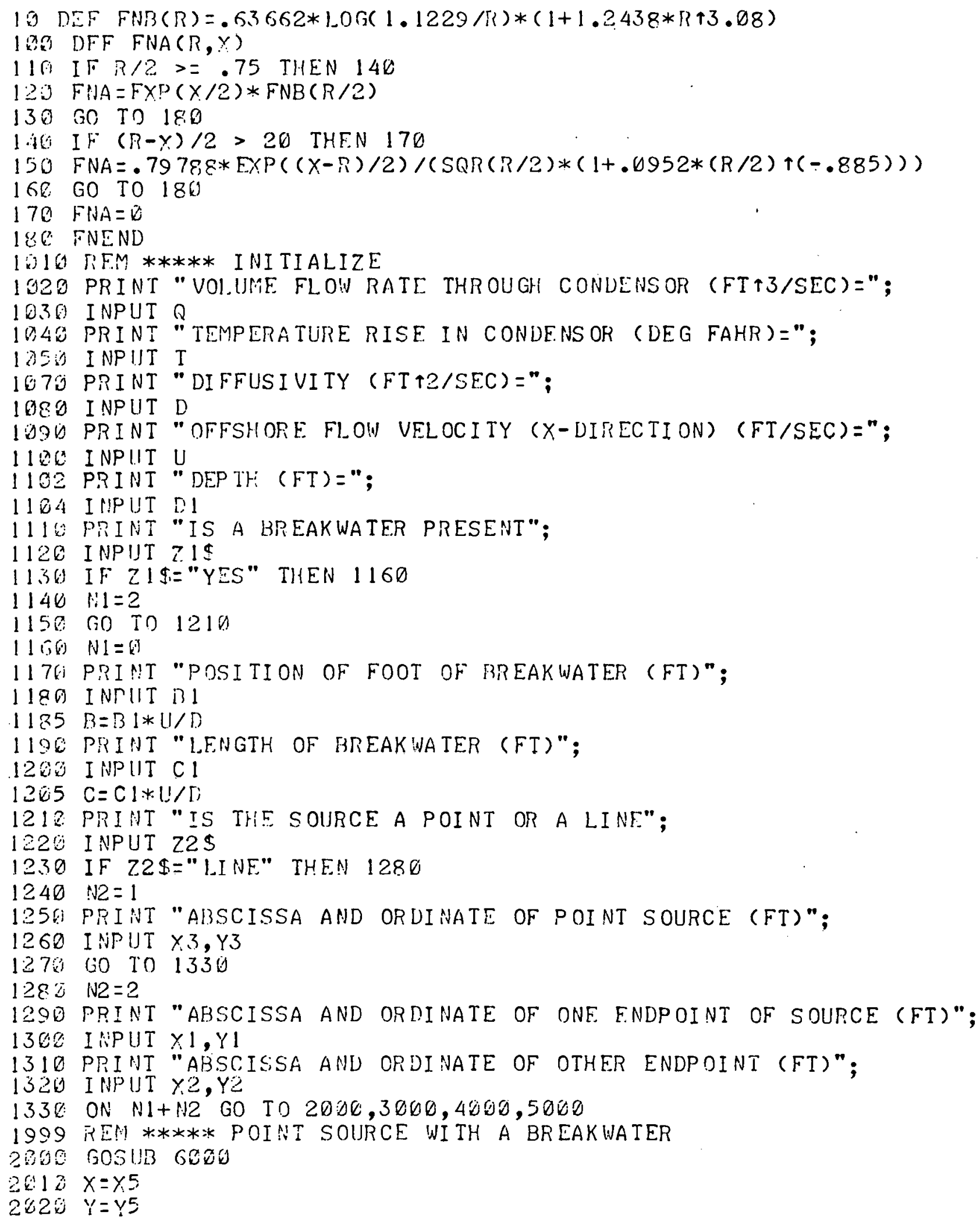


FARFLD

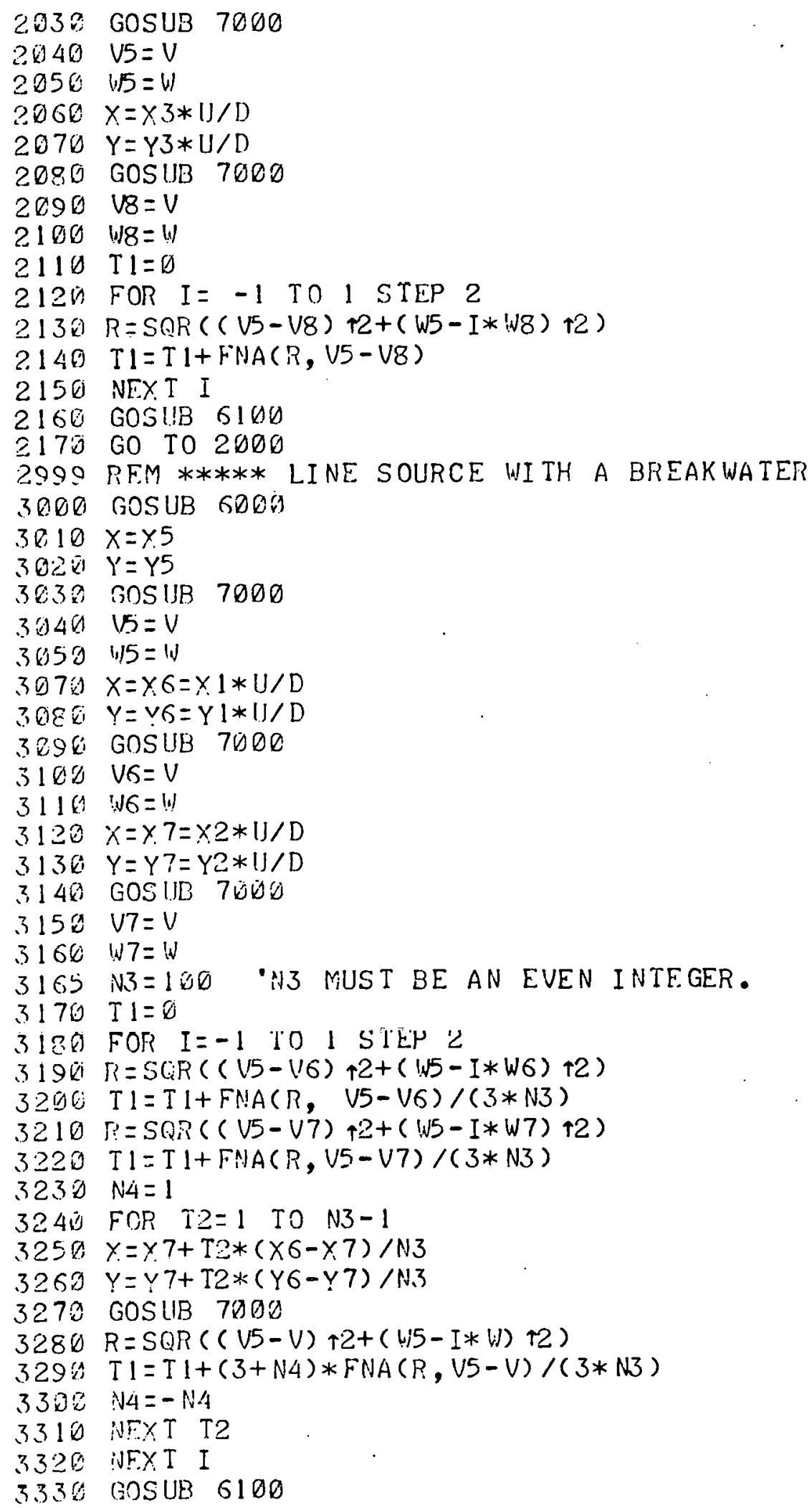


FARFLD

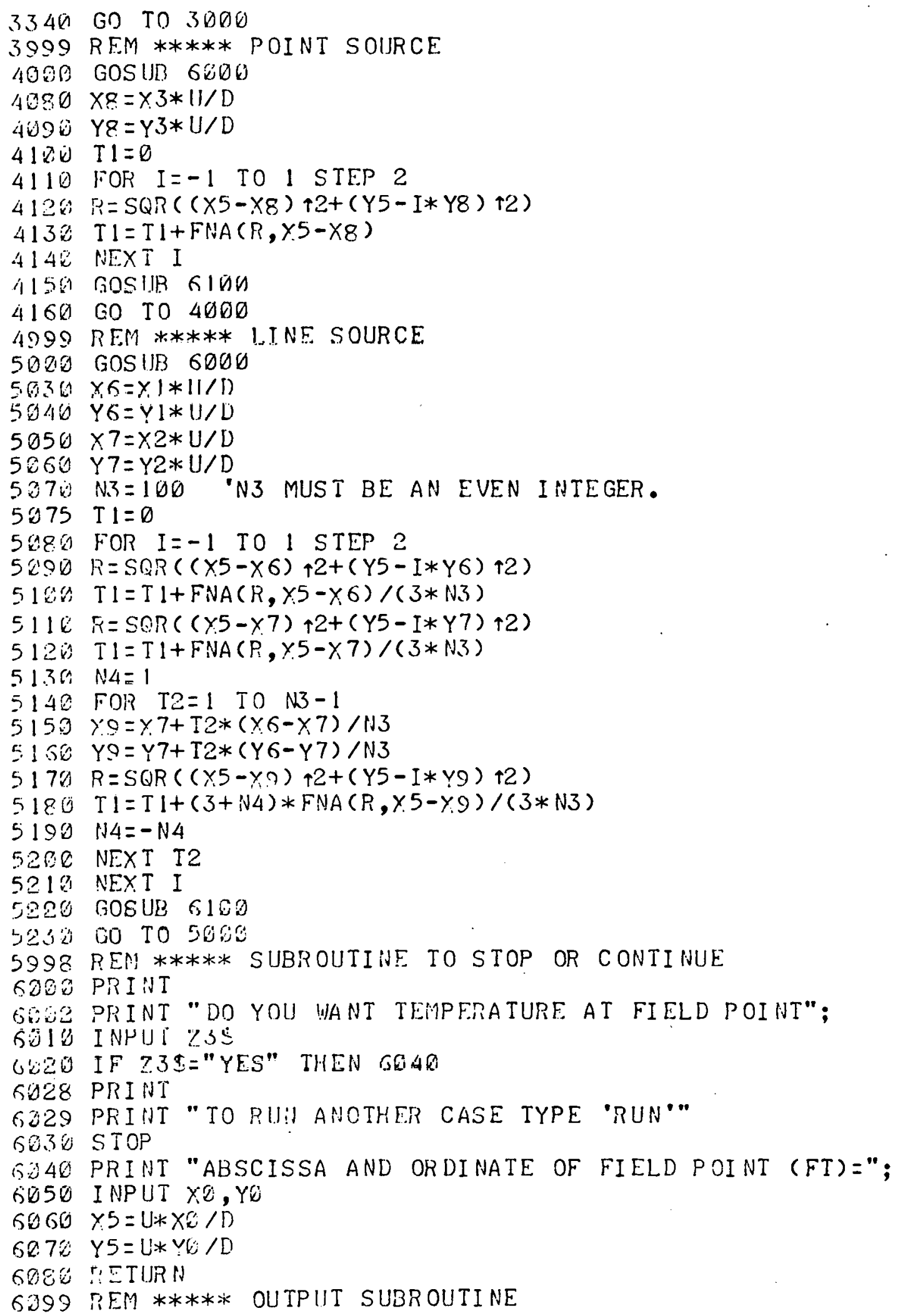


FARFLD

$6108, T 1=(\mathrm{Q} * \mathrm{~T} * \mathrm{~T} 1) /(4 * \mathrm{D} * \mathrm{D} 1)$

6110 PRINT "TEMPERATURE= "T1; "DF.GREES FAHRENHEIT"

6120 RETURN

6999 TEM ***** CONFORMAL MAPPING SUBROUTINE: $X, Y$ TO $V, W$

$7800 \quad A 1=(X-B) \uparrow 2-Y \uparrow Z+C \uparrow 2$

$7910 A 2=2 * Y *(X-B)$

$7020 \quad A .3=\operatorname{SS}(\Omega)(A 1+2 .+A 2 \uparrow 2)$

$703 * \quad V=S \operatorname{SGN}(A 2) * \operatorname{SQR}(.5 *(A 3+A 1))+\operatorname{SQR}(B+2+C \uparrow 2)$

$704 k i W=\operatorname{SinR}(.5 *(A 3-A 1))$

7050 RETURN

99993 END 
A typical conversation with the computer using the STRIP program. All entries typed by the user are underlined. Comments not a part of the actual conversation are typed in lower case letters.

G

$1 A C C / 50641 \quad 16: 29: 14$

PLEASE LOGIN OR ATTACH.

- LOGIN 6534,17

JOP 24 IACC/50641 TTY14

PASSWURU:

$1629 \quad 19-M A R-73 \quad$ MON

3-16-73 NE.W LOADER(56A) AND COMMAND INTERPRF. IQ

- $\underline{R}$ RASIC

j $-\left\{\begin{array}{l}\text { Message of the day-delete by } \\ \text { typing a control- } \varnothing .\end{array}\right.$

READY, FOR HELP TYPF HELP.

OLD STRIP

RFADY

RLIN

STRIP $\quad 16: 30 \quad 1 Y-M A R-73$

This initial message instructs the
usei liuw lu pruceed.

USFR: TYPE 'LISTNA 100-340. FOR MESSAGE. AFTFR MFSSAGE

SUIPPLY FUNCTIONS FNF $(X, Y)$ AND FNP $(X, Y)$ AND TYPE

-DELETE $10-40^{\circ}$. THEN TYPE 'RUN'.

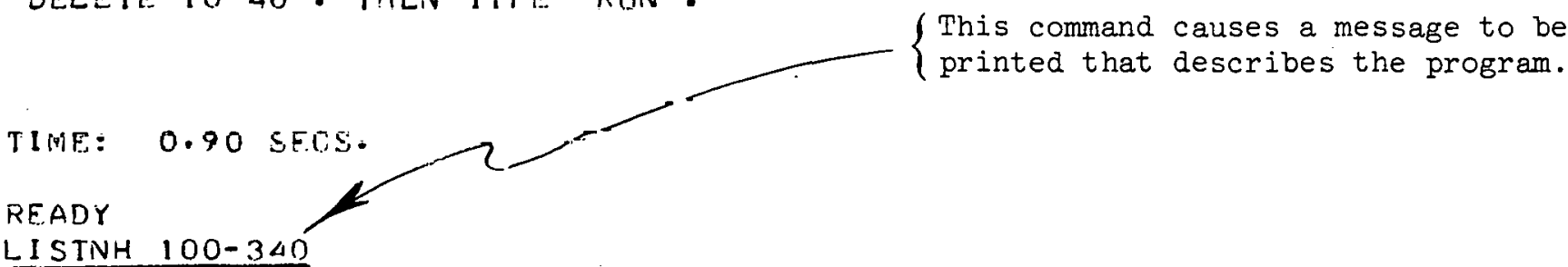

$100^{\circ}$ THIS PFOGRAM CALCULATES THETA AT ANY POINT IN AN ARFI TRARY THO-

$110^{\circ}$ DIMENSIONAL POTENTIAL FLOV FIELD. FGR HEAT DIFFUSION, THETA =

$120^{\circ} 4 * K * T / S$, WHERE $K=$ EDDY THERMAL COIVDUCIIVITY, T = TEMPERATURE.

$130^{\circ}$ RISE AROVE AMFIENT, AND $S=$ TOTAL SOURCE STRENGTH PER UNIT

$140^{\circ}$ DEPTH. THE DIMENSIONS OF THESE QUANTITIES ARE. AS FDLLGWS:

$150^{\circ}[K]=0 L i-1$ Ti-1 DEGT-1, [T] = DEG. AND [S] = O Li-1 Tr-1

$160^{\circ}$ (HERE $Q$ REPRESENTS HEAT). ANY UNITS GF LENGTH AND TIME MAY RE

$170^{\circ}$ USED, RUT THEY MUST RE USED CONSISTENTLY. FOR EXAMPLE, IF

$180^{\circ}$ LENGTHS ARE IN FEET AND TIME IS IN SECONDS, VELOCITIES MUST

$190^{\circ}$ RE GIVEN IN FEET PER SECOND AND DIFFUSIVITIES IN FEET SCUARED

$200^{\circ}$ PF.P. SFCOND, ETC. ANY UNITS DF HEAT AND TEMPERATURE MAY

$210^{\circ} P E$ USED, RUT THEY MUST ALSO RE USED CONSISTENTLY. 
2.0 - FOR THF DIFFUSION OF DISSOLVED SURSTANCES, THETA $=4 * A * C / S$, $230^{\circ}$ WHFRE $A=$ E.DDY DIFFUSIVITY, $C=$ CONCENTRATION, AND $S=$ TOTAL $240^{\circ}$ SOURCE STRENGTH PER UNIT DEPTH. THE DIMENSIGNS OF THESE 250. QUANTITIES ARE AS FOLLOWS: [A] = LI2. TI-1, [C] $=$ M LT-3 AND $260^{\circ}[S]=M L_{1-1} T+1$ (HERE M REPRESENTS AMOUNT). 270 AGAIN, ANY UNITS DF LENGTH AND TIME MAY PE USED AS LONG $280^{\circ}$ AS THEY ARE USED CONSISTENTLY. ANY UNITS MAY RE USED FDR 290.AMGUNT, E.G., MOLES, CURIES, GRAMS, F.TC. 300. THE SOURCE CAN BE EITHER A POINT OR A LINE. 310 THE USER MUST SUPPLY TWO FUNCTIONS, FNF(X,Y) AND FNP(X,Y), $320^{\circ}$ WHICH GIVE THE VELOCITY POTENTIAL ANO STREAM FUNCTION. RESPEC330.TIVELY, AT THE POINT $(X, Y)$. THESE FUNCTIONS GO IN LINES 340.1000-1999, RUT THE DEFINING LINES MUST USE LINES 1000 AND 1010.

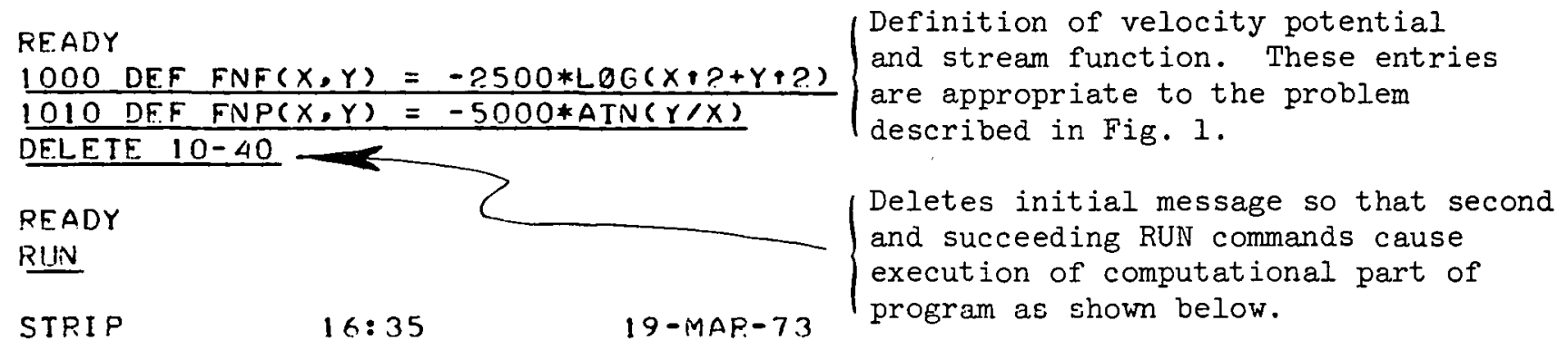

AFSCISSA AND ORDINATE OF A POINT ON ONE FDUNDARY ?5000,0 ABSCISSA AND GRDINATE OF A POINT ON OTHER BOUNDARY ? $5000,437.45$ DIFFUSIVITY $=$ ? 5 IS THE SOURCE A POINT OR A LINE ?POINT ARSCISSA AND ORDINATE OF SOURCE. ?

DO YOU WANT THETA AT FIELD POINT ?YES ARSCISSA AND ORDINATE OF FIELD POINT ? 5000,10 THETA $=0.53547$

DO YOII UANT THETA AT FIELD POINT ?YES AUSCISSA ANU ORUINATE OF FIELD POINT ? 4000.25 THETA $=0.144372$

DO YOU WIANT THETA AT FIELD POINT ?YES ARSCISSA AND ORDINATE OF FIELD POINT ?3000,100 THETA $=5.79259 E-2$

DO YOU WANT THETA AT FIFLD POINT ?NO TO RIIN ANGTHER CASE TYPE 'RUN' Z If a new velocity field is desired at this point, the functions FNF and FNP defined in lines 1000 and 1010 must be changed before typing "RUN."

TIME: 1.15 SECS \{Log off with this command.

\section{READY}

PYE

JOP. 24. USER [6534,17] LOGGED OFF TTY14 SAVED ALL 22. FILES (315. DISK BLOCKS)

RIINTIME O MIN, OS. ON SF.C

$1637 \quad 19-M A R-73$ 
A typical conversation with the computer using the FARFLD program.

- LOGIN $6534 / 17$

JOR ?.1 IACC/50641 TTY 14

PASSWORD:

$1638 \quad 19 \overline{9-M A R-73} \quad M O N$

- R RASIC

READY, FOR HELP TYPE HELP.

gLD FARFLD

RT:AIIY

RUN

FARFLD 19-MAR-73

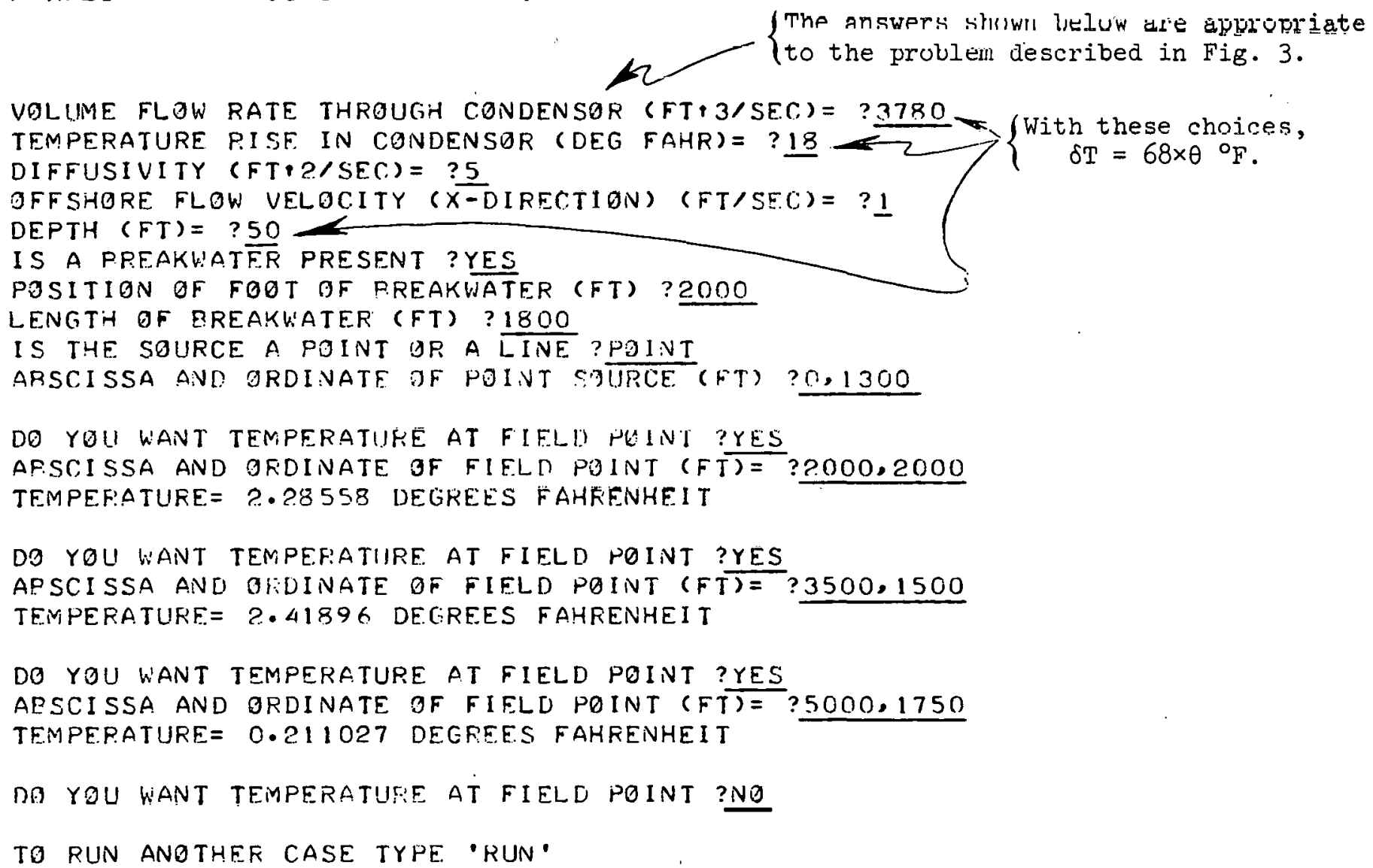

TIME: $\quad 1 \cdot 43 \quad \operatorname{SECS}$

READY

P.YE

JOR 21, USER [6534,17] LOGGED OFF TTY14, 1641 19-MAR-73

SAVED ALL 22 FILES ( 315 . DISK BLOCKS)

RUNTIME O MIN, 03.39 SEC 
I. S. E. Beall

2. F. T. Binford

3. W. E. Brow'ning

4. R. H. Bryan

5. R. H. Busey

6. V. R. Cain

7. W. B. Cottrell

8. C. C. Coutant

9. W. C. Cox

10. F. L. Culler

11. W. Davis, Jr.

12 16. L. Dresner

17. C. Easterly

18. A. Eraslan

19. A. P. Fraas

20. P. M. Garrett

21. C. P. Goodyear

22. J. A. Hafford

23. R. F. Hibbs

24. R. M. Hill

25. H. W. Hoffman

26. P. R. Kasten

27. G. W. Keilholtz

28. S. S. Kirslis

29. O. H. Klepper

30. M. I. Lundin

31. R. N. Lyon

32. R. E. McPherson

33. H. C. McCurdy

34. J. R. McWherter

35. A. J. Miller

36. M. Mitchell

37. D. J. Nelson

38. B. L. Nichols

39. R. B. Parker

40. A. M. Perry
41. E. Pickelsimer

42. D. A. Pilati

43. A. S. Quist

44. R. R. Rickard

45. R. C. Robertson

46. J. W. Roddy

47. M. W. Rosenthal

48 - 98. T. H. Row

99. R. M. Rush

100. T. Shapiro

101. M. R. Sheldon

102. S. Siegel

103. M. Siman-Tov

104. O. Sisman

105. M. J. Skinner

106. W. T. Snyder

107. I. Spiewak

108. W. G. Stockdale

109. E. G. Struxness

110. D. A. Sundberg

111. E. H. Taylor

112. R. E. Thoma

113. D. B. Trauger

114. W. C. Tunnell

115. F. Vaslow

116. A. M. Weinberg

117. G. D. Whitman

118-125. R. P. Wichner

126. M. M. Yarosh

127. W. C. Yee

128-130. Central Research Library

131. Document Reference Section

132-134. Laboratory Records Department

135. Laboratory Records - RC

136. EISO Library (Bldg. 2028)

137-153. Manager, Technical Information Center, AEC (ACRS Distribution)

154. Research and Technical Support Division, AEC, ORO

155. Patent Office, AEC, ORO

156-157. Technical Information Center, AEC 